\title{
Ueber die Bedeutung einer geordneten Milchkontrolle für die Städte.
}

Von

\author{
Dr. A. Lam.
}

Mittheilung aus dem städtischen Laboratorium v. d. Keuringsdienst van Voedingsmiddelen in Rotterdam.

Die nachfolgenden Zeilen zeigen, welche grosse Bedeutung abgesehen von der Aufdeckung verfälschter Milch eine geordnete Milchkontrolle für die Städte auch in der Richtung hat, dass durch dieselbe nicht nur die minderwerthige Waare möglichst beseitigt wird, sondern auch die Güte der Milch im Ganzen eine wesentliche Zunahme erfährt. In Rotterdam ist eine geordnete und regelmässige Milchkontrolle seit dem Jahre 1893 eingeführt und hat sich seit dieser Zeit sehr deutlich der günstige Einfluss der Kontrolle auf die Beschaffenheit der verkauften Milch erkennen lassen.

Die nachfolgende Uebersicht zeigt die Zahl der Beanstandungen in den Jahren 1893 bis 1899, so wie die relative Abnahme der beanstandeten Milchproben oder mit anderen Worten die relative Besserung in der Beschaffenheit der zum Verkaufe gelangten Milch von Jahr zu Jahr. Beanstandet wurde diejenige Milch, deren Trockensubstanzgehalt weniger als $11 \%$ und deren Fettgehalt weniger als $2,5 \%$ betrug.

\begin{tabular}{|c|c|c|c|c|c|c|c|}
\hline & 1893 & 1894 & 1895 & 1896 & 1897 & 1898 & 1899 \\
\hline Beanstandete Milch \% & $49,0 \%$ & $20,3 \%$ & $11,9 \%$ & $5,3 \%$ & $3,3 \%$ & $3,7 \%$ & $3,8 \%$ \\
\hline $\begin{array}{l}\text { Procentige Abnahme der } \\
\text { Beanstandungen von Jahr } \\
\text { zu Jahr }\end{array}$ & & & & & & & \\
\hline
\end{tabular}

Seit dem Jabre 1897 scheint somit das durch eine geordnete Milchkontrolle überhaupt erreichbare Minimum der Beanstandungen erlangt zu sein. In Wirklichkeit ist die Beschaffenheit der Milch aber auch seit dem Jahre 1897 noch eine bessere geworden. Bis zum Jahre 1897 wurden die Milchproben ohne besondere Auswahl überall dort genommen, wo überhaupt ein Milchverkauf stattfand; in den Jahren 1898 und 1899 dagegen waren die Inspektoren der Nahrungsmittelkontrolle angewiesen, hauptsächlich Proben an den Stellen zu entnehmen, bei denen der Verdacht vorhanden war, dass sie dann und wann verfälschte oder minderwerthige Milch lieferten.

Es ist einleuchtend, dass in diesem Falle der Procentsatz von zu beanstandender Milch höher sein wird, als wenn die Untersuchung sich ohne Auswahl auf alle zum Verkaufe gelangende Milch beziehen würde. Die Besserung in der Beschaffenheit der Milch ergiebt sich aber auch noch auf andere Weise. Wenn die gefälschte bezw. minderwerthige Milch in der That mehr und mehr verschwunden ist, dann muss auch der mittlere Gehalt der Milch seit 
dem Jahre 1893 ein höherer geworden sein. In der That ist dies der Fall. Wenn man z. B. den Fettgehalt der Milch in den Monaten September bis December 1893 und 1899 vergleicht, so ergeben die 150 bis 180 monatlichen Untersuchungen folgenden mittleren Fettgehalt.

\begin{tabular}{ccccc} 
Jahr & September & October & November & December \\
1893 & $2,34 \%$ & $2,40 \%$ & $2,67 \%$ & $2,69 \%$ \\
1899 & $3,17-$ & $3,15-$ & $3,21-$ & $3,28-$ \\
\hline Also im Jahre & $0,83 \%$ & $0,75 \%$ & $0,54 \%$ & $0,59 \%$ \\
1899 mehr Fett & &
\end{tabular}

Es ist also eine recht erfreuliche Besserung in der Beschaffenheit der Milch zu erkennen, deren wirthschaftliche Bedeutung nicht zu unterschätzen ist.

Wenn man berücksichtigt, dass der tägliche Milchverbrauch in Rotterdam nahezu 100000 l beträgt, so besagt das obige Ergebniss, dass in Rotterdam z. B. im September 1899 täglich über $\left.{ }^{1}\right) 100000 \times 8,3 \mathrm{~g}=830 \mathrm{~kg}$ Milchfett mehr verabreicht werden, als im Jahre 1893. Diese Milchfettmenge reicht aus zur Herstellung von mehr als $800 \mathrm{~kg}$ Butter im Werthe von 630 Holl. Gulden oder von mehr als 1050 M. Diesem täglichen Mehrwerth der Milch entspricht ein jährlicher Gewinn von 230000 Holl. Gulden oder 383000 M. Der wirthschaftliche und ebenso der Nährwerth der Milch haben mithin seit dem Jahre 1893 eine bedeutende Zunahme erfahren, denn der Preis der Milch ist nach wie vor derselbe geblieben.

Seit dem Jahre 1895 sind seitens des hiesigen Laboratoriums regelmässig, wöchentlich einmal bei drei bis sieben verschiedenen Viehhaltern Milchproben entnommen worden. Die Milch wurde im Beisein eines Laboratoriumsdieners, der auf Reinheit der Milchgefässe und sonstige Vorsichtsmaassregeln zu achten hatte, gemolken und von dem Diener selbst nach wiederholtem Mischen der Milch die Probe entnommen. Auf diese Weise wurde eine grosse Zahl zuverlässig entnommener Proben der Mischmilch einer grösseren Anzahl von Kühen verschiedener Herkunft gewonnen. Die Untersuchung dieser Proben „Kontrollmilch" führte zu Mittelwerthen, die in den einzelnen Jahren nur wenig schwankten. Es wurden für die „Kontrollmilch“ folgende Mittelwerthe gefunden:

\begin{tabular}{c|c|c|c|c|c|c}
\hline Jahr & $\begin{array}{c}\text { Spec. Gewicht } \\
\text { bei 150 C. }\end{array}$ & Trockensubstanz & Fett & $\begin{array}{c}\text { Fettfreie } \\
\text { Trockensubstanz } \\
\%\end{array}$ & $\begin{array}{c}\text { Fett in der } \\
\text { Troekensubstanz } \\
\%\end{array}$ & $\begin{array}{c}\text { Milehzucker } \\
\text { (polarimetriseh } \\
\text { bestimmt) } \\
\%\end{array}$ \\
\hline 1895 & 1,0313 & $\mathbf{1 2 , 1 0}$ & 3,50 & 8,60 & 28,9 & - \\
1896 & 1,0314 & $\mathbf{1 2 , 1 6}$ & $\mathbf{3 , 5 4}$ & 8,62 & 29,1 & 4,89 \\
1897 & 1,0314 & $\mathbf{1 2 , 2 5}$ & $\mathbf{3 , 6 4}$ & 8,61 & 29,7 & 4,97 \\
1898 & 1,0316 & $\mathbf{1 2 , 2 9}$ & $\mathbf{3 , 6 2}$ & 8,67 & 29,5 & 4,77 \\
1899 & $\mathbf{1 , 0 3 1 3}$ & $\mathbf{1 2 , 3 3}$ & $\mathbf{3 , 6 5}$ & 8,68 & 29,6 & 4,82
\end{tabular}

Auch die Höchst- und Niedrigstwerthe für die Bestandtheile der einzelnen Milchproben schwanken in den versehiedenen Jahren nur wenig, so dass man

1) Da 11 Milch durchschnittlich 1,03 kg wiegt. 
die vorstehenden Zahlen als mittlere Zusammensetzung der Milch der hiesigen Gegend ansehen kann.

Vergleicht man nun den mittleren Fettgehalt der "Kontrollmilch" mit dem oben mitgetheilten mittleren Fettgehalt, welcher bei der verkauften Mileh in den einzelnen Monaten ermittelt wurde, so ersieht man, dass der Fettgehalt der verkauften Milch wesentlich niedriger ist als der der "Kontrollmilch“-Proben. Diese Erscheinung kann darin begründet sein, dass entweder die Verkaufsmilch noch in hohem Maasse gefälscht wird, oder aber die untersuchte Verkaufsmilch ist von Hause aus minderwerthiger als die Kontrollmilch $d$. h. die untersuchten Verkaufsmilchproben sind keine wirklichen Durchschnittsproben aller zum Verkaufe gelangenden Milchsorten. Letzteres ist in der That der Fall. Denn, wie ich oben schon ausführte, werden bei der Kontrolle der Marktmilch die Proben hauptsächlich bei solchen Verkäufern entnommen, von denen bekannt ist, dass sie eine minderwerthige Milch liefern. Es bleibt aber noch immer die Möglichkeit bestehen, dass die Milch ausserdem noch in merklichem Maasse gefälscht wird.

Um hierüber näheren Aufschluss zu erhalten, liegt es nahe, den Fettgehalt der Kontrollmilch und den der Verkaufsmilch in den einzelnen Monaten des Jahres zu vergleichen. Aus Gründen der inneren Einrichtung der hiesigen Nahrungsmittel-Kontrolle habe ich diesen Vergleich erst im September 1898 begonnen und ihn bis Ende 1899 fortgesetzt. Die nachfolgende Tabelle zeigt die entsprechenden Fettgehalte von Kontrollmilch und Marktmilch in den einzelnen Monaten. Es betrug der mittlere procentige Fettgehalt:

\begin{tabular}{|c|c|c|c|c|c|c|c|c|c|c|c|c|c|c|c|c|c|}
\hline & \multicolumn{4}{|c|}{1898} & \multicolumn{12}{|c|}{$\underbrace{1899}$} & \multirow[b]{2}{*}{$\mid \begin{array}{l}\text { 䓌 } \\
\text { 荧 }\end{array}$} \\
\hline & 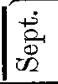 & 离 & 官 & $\dot{\dot{\Phi}}$ & 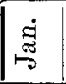 & $\mid \begin{array}{l}\dot{\Delta} \\
\stackrel{0}{0} \\
\dot{0}\end{array}$ & 㥑 & $|\overline{\bar{z}}|$ & 茜 & 害 & 莺 & 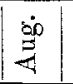 & $\left|\begin{array}{c}\dot{\overrightarrow{2}} \\
\vec{D}\end{array}\right|$ & $\dot{\vec{y}}$ & 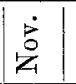 & $\mid \begin{array}{c}\dot{\Phi} \\
\dot{\Phi}\end{array}$ & \\
\hline Kontrollmilch & 3,62 & 3,68 & 3,69 & 3,55 & 3,55 & 3,60 & 3,64 & 3,75 & 3,70 & 3,52 & 3,61 & 3,56 & 3,77 & 3,64 & 3,66 & 3,77 & 3,65 \\
\hline Marktmileh & 3,11 & 3,18 & 3,21 & $3,14 \mid$ & $3,13 \mid$ & 3,14 & 3,17 & 3,22 & 3,14 & 3,02 & 3,04 & 3,11 & $3,17 \mid$ & 3,15 & 3,21 & 3,28 & 3,15 \\
\hline Differenz & 0,51 & & & 0,41 & & & & 0,5 & & 0,5 & & & 0,0 & 9 & 0,4 & 0,49 & 0,50 \\
\hline
\end{tabular}

Wie man aus dieser Zusammenstellung ersieht, gehen der Fettgehalt der Kontrollmilch und der der Marktmilch in den einzelnen Monaten nahezu vollständig parallel. Noch besser erhellt dieser Parallelismus aus der graphischen Darstellung (Fig. 28) auf S. 475.

Beim Vergleich der obigen Zahlen und der nachstehenden Kurven für den Fettgehalt der Kontrollmilch und der Marktmilch kommt man zu der Ueberzeugung, dass im Grossen und Ganzen die Milchfälschungen nicht die Ursache der Minderwerthigkeit der Marktmilch gegenüber der Kontrollmileh sind. Denn, wenn gleiche Erfolge auf gleiche Ursachen hinweisen, so kann auch der unzweifelhafte Parallelismus zwischen dem Fettgehalt der Kontrollmilch und Marktmilch in den einzelnen Monaten des Jahres nur durch die gleichen Umstände verursacht sein. Da aber bei der Kontrollmilch Fälschungen bestimmt 
ausgeschlossen sind, so wird man auch annehmen müssen, dass Fälschungen auch bei der Marktmilch keinen erheblichen Einfluss auf die mittlere Zusammensetzung der Milch gehabt haben.

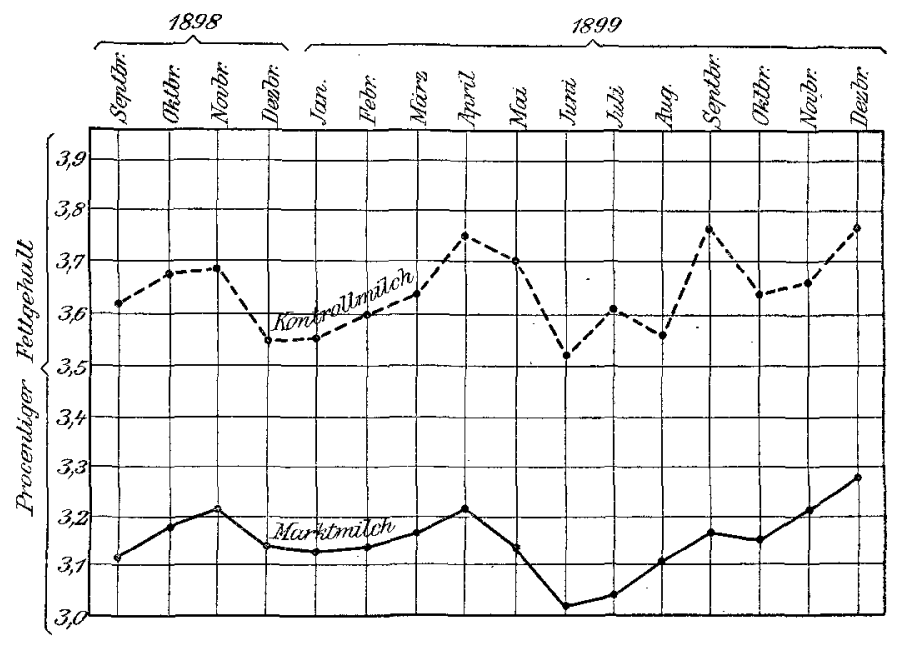

Fig. 28.

Die vorstehenden Zeilen zeigen, wie man durch Vergleich einer genügenden Zahl von Kontrollmilch- und Marktmilchproben einen Einblick in den Erfolg der Milchkontrolle erhält.

\section{Referate.}

\section{Fleisch, Fleischwaaren und diätetische Nährmittel.}

Franz Lehmann: Ueber die Qualität des Karpfenfleisches. - Allgem. Fischerei-Ztg. 1900, 25, 91-96.

Die Untersuchung des Fleisches von Karpfen, die in verschiedener Weise gefüttert worden waren, ergab folgende Werthe:

\begin{tabular}{l|c|c|c|c}
\hline \multirow{2}{*}{ Best a n d t h e i l e. } & \multicolumn{4}{|c}{ Die Karpfen wurden gefüttert mit } \\
& $\begin{array}{c}\text { Fleischmehl } \\
\text { und Mais } \\
\%\end{array}$ & $\begin{array}{c}\text { Mais und } \\
\text { Lupinen } \\
\%\end{array}$ & $\begin{array}{c}\text { Lupinen } \\
\%\end{array}$ & $\begin{array}{c}\text { Nicht } \\
\text { gefuttert } \\
\%\end{array}$ \\
\hline Wasser & 73,89 & 71,60 & $\mathbf{7 4 , 9 2}$ & $\mathbf{7 8 , 8 5}$ \\
Trockensubstanz & 26,11 & 28,40 & 25,08 & 21,15 \\
Stickstoffsubstanz & 16,73 & 16,17 & 17,11 & $\mathbf{1 7 , 3 8}$ \\
Fett & 8,34 & 11,13 & 6,82 & 2,57 \\
Asche & $\mathbf{1 , 1 3}$ & $\mathbf{1 , 1 2}$ & 1,16 & 1,22 \\
Stickstoff & 2,94 & 2,68 & 2,84 & 2,91 \\
\hline Ausbeute an Fleisch & 45,3 & 45,8 & 46,6 & 45,3 \\
Jodzahl des Fettes & 72,1 & 99,2 & 83,2 & 87,9
\end{tabular}

*) Die Stickstoffsubstanz ist aus der Differenz: Trockensubstanz minus (Fett und Asche, berechnet. 